\title{
Sensitivity of Aerofoil Self Noise Reductions to Serration Flap Angles
}

\author{
A. Vathylakis ${ }^{1}$, T. P. Chong ${ }^{2}$, C. Paruchuri ${ }^{3}$ and P. F. Joseph ${ }^{4}$ \\ ${ }^{1-2}$ Brunel University London, Uxbridge, UB8 $3 P H, U K$ \\ ${ }^{3-4}$ University of Southampton, Southampton, SO17 1BJ, UK
}

\begin{abstract}
The serration amplitude and serration wavelength are traditionally regarded as the primary geometrical variables that can affect the noise performance of an add-on, flat plate type serrated trailing edge. This experimental study investigates another serration geometrical variable, namely the serration flap angle that could potentially affect the self-noise reduction of an aerofoil. The experiment was carried out at Brunel aeroacoustic facility, on a NACA65(12)-10 aerofoil. The serrated flat plates were manufactured to form in several flap angles: $\pm 15^{\circ}, \pm 10^{\circ}, \pm 5^{\circ}$ and $0^{\circ}$ as the reference. Preliminary investigation on the effect of serration amplitude, without the flap angle, confirms with other findings that the largest level of broadband noise reduction is achieved when the amplitude of the serrated flat plate is large. It is also worth reporting that broadband noise can already be reduced even by attaching a large chord length of unserrated, straight flat plate. When the serrated flat plate contains a flap angle, it is generally observed that a flap-up position (positive flap angle) is more favourable for broadband noise reduction, while the opposite is true for the flap-down position (negative flap angle). The best flap-up position is when the positive flap angle is small, at around $+5^{\circ}$. Unfortunately, a small flap-down position, i.e. $-5^{\circ}$ is the worst performer amongst the test cases (lowest level of broadband noise reduction at low frequency, and highest noise increase at high frequency). Therefore, even a small misalignment of the trailing edge serration due to the manufacturing defect could potentially degrade (or enhance) the overall aerofoil self-noise reduction because the serration is found to be sensitive to small flap angles.
\end{abstract}

\section{Introduction}

Self-noise emitted from the trailing edge of an aerofoil blade represents a major environmental and operational Sissue in aviation, wind turbine and home appliance industries. There has been much interest recently in developing flow control methods aimed at reducing trailing edge self-noise. For example, active flow control of wall-normal suction was implemented in wind turbine blades to reduce trailing edge noise ${ }^{1}$. Another active flow control method for the suppression of trailing edge self-noise is achieved by the Dielectric Barrier Discharge plasma actuators ${ }^{2}$. In this case, the induced air jet by the actuators can disrupt the growth of the boundary layer instabilities, thus resulting in the suppression of instability tonal noise. In terms of aerofoil self-noise reduction by passive flow control, one of the most commonly used methods is inspired by the owl's wing. The unique feature of trailing edge serration is known to be quite effective in reducing both aerodynamic drag $^{3,4}$ and self-noise radiation ${ }^{5-11}$.

\footnotetext{
${ }^{1}$ Research Associate, Department of Mechanical, Aerospace and Civil Engineering, alex.vat@hotmail.com, Non-AIAA Member (corresponding author)

${ }^{2}$ Senior Lecturer, Department of Mechanical, Aerospace and Civil Engineering, t.p.chong@ brunel.ac.uk, AIAA Member

${ }^{3} \mathrm{PhD}$ student, Institute of Sound and Vibration Research, c.c.paruchuri@ soton.ac.uk, Student Member AIAA

${ }^{4}$ Professor, Institute of Sound and Vibration Research, pfj@soton.ac.uk. Senior Member AIAA
} 
In the case of a fully turbulent boundary layer, for example at high Reynolds numbers, or when tripping is applied, some of the turbulent energy in the boundary layer will be scattered into broadband noise at the trailing edge. Typical broadband noise amplitudes are considerably lower than the tonal noise resulting from boundary layer instability. The relationship between the far field acoustic pressure and the near field surface pressure near the trailing edge is made explicit in the classical work of Amiet ${ }^{12}$ who derived a direct relationship between the power spectral density of the far field noise $S_{p p}$ in terms of the spanwise correlation length $I_{y}$ and the surface pressure spectrum $S_{q q}$ near the trailing edge, and a radiation term $L(\omega)$, of the form: $S_{p p}(\omega) \propto L(\omega) I_{y}(\omega) S_{q q}(\omega)$. This result predicts a reduction in the radiated broadband noise if the level of either $I_{y}, S_{q q}$ and/or $L(\omega)$ is reduced. A sawtooth surface has the potential to modify one or more of the above three source terms, possibly leading to a reduction of the radiated noise.

A comprehensive experimental study by Gruber et al. ${ }^{9}$ on many sawtooth geometries has established that significant noise reduction can be achieved if two conditions are fulfilled. The first is when the serration length is of the same order as the turbulent boundary layer thickness near the trailing edge. The second is when the serration angle is small, giving the appearance of a sharp sawtooth. These conditions generally agree well with the recommendation given by Howe ${ }^{6}$. The same observation was also reported by Chong et al. who investigated several nonflat plate type serrated trailing edges ${ }^{10}$.

The noise performance of a serrated trailing edge can also be influenced by a third sawtooth geometrical variable, which has not yet received much attention. This geometrical variable is the flap angle $(\phi)$, or inclination angle relative to the aerofoil camber line of the serrated flat plate, as illustrated in Fig. 1 on a NACA65(12)-10 aerofoil. When the serration flat plate, or element of the sawtooth is deflected "upward" with relative to the zero flap angle, a positive flap angle $\phi$ is produced. Likewise, a "downward" deflection of the serration flat plate will produce a negative $\phi$. This sign convention is adopted throughout the paper here. As will be shown later, varying the flap angle of the serrated flat plate can produce considerably different noise performances. This has considerable ramification for industrial blades, e.g. the wind turbine, that adopt serration technology because the noise performance can be very susceptible for misalignment between the incoming flow angle and the serration flap angle.

The change in noise characteristics could be attributed to two possible mechanisms. The first is related to the change in global flow field around the aerofoil when introducing a flap angle to the serrated flat plate. The different blade loading will almost certainly affect the growth of boundary layers, thereby resulting in different self-noise characteristics. The second mechanism is considered as more localised. It is anticipated that introducing a flap angle to the serrated flat plate will encourage three-dimensionality flow fields at region close to the trailing edge. Gruber et al. ${ }^{9}$ visualised the cross-jet across the sawtooth gaps, where they suggested that the interaction between the crossjet and the sawtooth geometry could cause high frequency noise increase. The cross-jet across the sawtooth gaps is also inferred by Chong and Vathylakis ${ }^{13}$ as a source to trigger the vortical structures at the vicinity of the oblique edge to interact with the local turbulent boundary layer. Such viscous and inviscid interaction will re-distribute the momentum and turbulent shear stresses along the sawtooth edges and tips, and reduce the acoustical-scattering efficiency of the hydrodynamic pressure fluctuation into noise. A serrated flat plate with flap angle is likely to affect the cross-jet and the resulting self-noise characteristics.

Aerodynamically, adding a flap angle at the trailing edge of an aerofoil blade will also produce different levels of lift and drag coefficients. For an aircraft wing, a down-deflected flap $(-\phi)$ will normally cause an increase in lift coefficient, as well as increase in drag coefficient. An upward deflection of flap $(+\phi)$ can distort the aerofoil streamline due to the trailing edge cusp. Therefore, it is expected that less lift is produced, but the drag will be significantly increased.

Therefore, the flap angle of the serrated flat plate is an important geometrical variable for the serration technology, but it has largely been overlooked so far. The main objective of this paper is to perform a preliminary study to investigate the overall performance of serrated trailing edge with different flap angles, and their effect on the boundary layer near the trailing edge and the near wake velocity.

\section{Experimental setup}

\section{A. Design of aerofoil and trailing edge serration with flap angles}

The aerofoil model used in this study is an NACA65(12)-10 with $0.15 \mathrm{~m}$ chord, $C$ and $0.45 \mathrm{~m}$ span. This particular aerofoil model, as well as the magazine of straight (baseline) and serrated flat plates, is the same one used in the previous study at Institute of Sound and Vibration Research (ISVR), University of Southampton'. This particular type of aerofoil can be found in the compressor stage of some engines. Figure 1 shows a photograph of the 
aerofoil and several sketches to show the definitions of: serration amplitude $(2 h)$, serration wavelength $(\lambda)$ and serration flap angle $(\phi)$. In order to focus on the effects of flap angle and serration amplitude to the self-noise radiation, the serration wavelength was kept the same at $\lambda=3.3 \mathrm{~mm}$ throughout the experiment. Note that a coordinate system is also defined: streamwise $(x)$, vertical $(y)$ and spanwise $(z)$.

The aerofoil is composed of a main body and a detachable trailing edge, which allows flat plate serration geometries to be inserted at the aerofoil trailing edge. As shown in Fig. 1, trailing edge serrations are cut into thin flat plates and inserted into the rear part of the aerofoil. For the flat plates (both straight and serrated) designated to have zero degree flap angle $\phi=0^{\circ}$, they are made of cardboard of approximately $0.8 \mathrm{~mm}$ thick. For non-zero flat angles $\phi \neq 0^{\circ}$, the straight and serrated flat plates were 3D-printed so that a desired flap angle can be accurately preformed. The thickness of these 3D-printed flat plates is also $0.8 \mathrm{~mm}$. Zig-zag glue-on tapes were applied at both the suction and pressure surfaces near the aerofoil's leading edge in order to trip the boundary layer into turbulent. This is to ensure that turbulent noise sources can be generated at the trailing edge.

\section{B. Wind tunnel facilities and instrumentation}

All the free field measurements of the aerofoil self noise were conducted in the aeroacoustic facility at Brunel University London. The open jet wind tunnel is situated in a $4 \mathrm{~m}$ x $5 \mathrm{~m}$ x $3.4 \mathrm{~m}$ hemi-anechoic chamber. The nozzle exit is rectangular with dimensions of $0.10 \mathrm{~m}$ (height) $\mathrm{x} 0.30 \mathrm{~m}$ (width). The jet velocity $(U)$ can reach $80 \mathrm{~ms}^{-1}$, but in the current run we only tested up to $U=60 \mathrm{~ms}^{-1}$. The background noise of the wind tunnel facility is well below the self-noise of the quietest aerofoil at the lowest velocity. Most of the results presented in this paper correspond to a flow case when $U=24 \mathrm{~ms}^{-1}$, although there is one case where a range of jet speeds $20 \leq U \leq 60 \mathrm{~ms}^{-1}$ was also investigated. The aerofoil was held by side plates and attached flushed to the nozzle lips. In this study, the angle of attack (AoA) was adjusted to zero degree only.

As shown in Fig. 2, far field noise measurements at the aeroacoustic wind tunnel were made by a condenser microphone at polar angles of $\Theta=90^{\circ}$ at a distance of $1.0 \mathrm{~m}$ from the aerofoil at mid span. Noise data was acquired at a sampling frequency of $44 \mathrm{kHz}$ for $20 \mathrm{~s}$ by a 16-bit Analogue-Digital card from National Instrument. The data was then windowed and the Power Spectral Density $(P S D)$ of $1 \mathrm{~Hz}$ bandwidth computed from a 1024 point FFT.

A single hot wire probe ( $5 \mu \mathrm{m}$ diameter, $1.25 \mathrm{~mm}$ length, DANTEC 55P11) was used to investigate the upstream boundary layer mean and fluctuating velocity profiles subjected to the straight and serrated flat plates. The hot wire was operated at an overheat ratio of about 1.8 , which has a good velocity sensitivity. The near wake velocity power spectral densities and coherence function in the spanwise direction were also investigated by two single hot wire probes - one is termed as a "stationary probe", and another one is called "traversing probe". More details about their operating procedure will be discussed in Section IV.B. The overheat ratios in this case were adjusted to a slightly lower value of 1.6. This is to minimise thermal interference when the two probes are in close proximity to each other when performing the coherence measurement. Signals from these hot wire probes were digitised by a 12-bit A/D converter (TSI model ADCPCI) at a sampling frequency of $20 \mathrm{kHz}$ for 120000 realisations. The hot wire probe was attached to a computer-controlled two-dimensional traverse system with a resolution of $0.01 \mathrm{~mm}$ in both directions.

\section{Serrated Trailing Edge with Zero Flap Angle}

This section will first discuss the aerofoil self-noise and its reduction by add-on serrated flat plates at zero flap angle, $\phi=0^{\circ}$. It is important to note that the quantification of noise reduction by the serrated flat plate is measured against a straight, unserrated flat plate with the same wetted area. This approach is also adopted by Gruber et $\mathrm{al}^{9}$ to ensure a same planform area for both the serrated and baseline aerofoils. Essentially, a serrated flat plate that comprises a uniform sawtooth length of $H$ will be compared against a straight flat plate of length $H / 2$. This definition is adopted throughout this paper.

The NACA65(12)-10 model and the serrated flat plates tested here were previously used by Gruber et al. ${ }^{9}$ in the ISVR, Southampton. It would be important to ensure that the serrated aerofoil self-noise measured in the Brunel aeroacoustic facility has the same characteristics as Gruber et al. Figure 3 compares the noise spectra produced by a same type of serrated aerofoil $(\lambda / h=0.2)$ at $\mathrm{AoA}=0^{\circ}$ between the current setting and Gruber et al.'s. Note that because a single point measurement was made in this study, the comparison with Gruber et al., who mostly presented the results in sound power, can only be made qualitatively. From the figure, a reasonably well match in the noise reduction (or increase) for several combinations of frequency and velocity ranges occurs. Generally speaking, a serrated aerofoil in the flat plate configuration can cause a reduction in broadband noise at low frequency, but can also cause an increase of noise at high frequency. The threshold that separates these two regions is found when $f \delta_{t u r} / U \approx 1$, where $f$ is the frequency and $\delta_{t u r}$ is the turbulent boundary layer thickness near the trailing 
edge (the $\delta_{t u r}$ was estimated numerically in Gruber et al.). The reduction in broadband noise can be attributed to the serration effect. The noise increase at high frequency, however, is likely to be caused by the interaction between the cross-jet and the sawtooth gaps as suggested by Gruber et $\mathrm{al}^{9}$.

\section{A. Effect of flat plate length on the self noise radiation at $U=24 \mathrm{~ms}^{-1}$}

Before the serrated flat plates are compared against the baseline, straight flat plates, this sub-section will first investigate the effect of the flat plate length $(2 h)$ for the aerofoil self-noise, individually. In the current study, we have chosen the range of $2 h$ to be between 2 and $35 \mathrm{~mm}$ for both the straight and serrated flat plates. Figure $4 \mathrm{a}$ shows the collection of noise spectra for the straight flat plate at $U=24 \mathrm{~ms}^{-1}$, AoA $=0^{\circ}$ and $\phi=0^{\circ}$. Interestingly, it has been demonstrated that aerofoil self-noise reduction can already be achieved at $250 \leq f \leq 2500 \mathrm{~Hz}$ simply by adding straight flat plate inserts to the aerofoil. The level of reduction is found to increase as $2 h$ increases. In contrast, at frequency range of $2.5<f \leq 5.0 \mathrm{kHz}$, noise increase occurs as the $2 h$ increases. This point is intriguing because the straight flat plates, which do not have any sawtooth air gaps, are unlikely to facilitate any interaction with the cross-jet, if any.

In general, increasing $2 h$ will cause a reduction of noise at low frequency, but increase of noise at high frequency. This trend suggests that the self-noise is a function of the boundary layer properties near the flat plate edge. However, as one would expect the boundary layer to become thicker when $2 h$ increases, the increase in size of the largest eddy scale in the boundary layer would normally translate into increase of noise level at low frequency and reduction in high frequency noise levels. This reasoning, however, contradicts the measured noise spectra in Fig. 4a. This implies that the change in noise characteristics simply by adding straight flat plate is more related to the change in blade loading. We conjecture that the boundary layers at the suction and pressure surfaces may not respond in the same way to the change in overall blade loading. Therefore, it is possible that the combined eddy size from the suction and pressure surfaces at the vicinity of the trailing edge is actually smaller as $2 h$ increases. Further investigation is certainly needed to verify this conjecture.

Figure $4 \mathrm{~b}$ shows the collection of noise spectra for the serrated flat plate also at $U=24 \mathrm{~ms}^{-1}, \mathrm{AoA}=0^{\circ}$ and $\phi=$ $0^{\circ}$. For consistency, the serration wavelength, $\lambda=3.3 \mathrm{~mm}$ for all the cases. The same trend described above can also be observed, but with several minor differences. Increasing the $2 h$ for the serrated flat plate slightly pushes the noise reduction bandwidth towards the low frequency end: $100 \leq f \leq 2000 \mathrm{~Hz}$. It is interesting to note that, at $100 \mathrm{~Hz}$, increasing the $2 h$ of the serrated flat plate can reduce the noise in a consistent manner. This implies that the serrated flat plate has a larger effect on the blade loading. Generally, the level of noise reduction as a function of $2 h$, compared to the straight flat plates, is much larger for the serrated flat plates.

The radiated noise level is also found to increase as a function of $2 h$ at the high frequency, $f \geq 2.5 \mathrm{kHz}$. However, the frequencies that mark the start of the noise increase are almost constant for all the serrated flat plates (different $2 h$ ). Therefore, the noise source is unlikely to be related to the sawtooth length per se. It is important to point out that all the noise spectra in Fig. $4 \mathrm{~b}$ are produced by serrated flat plates, including the reference spectrum (black line) where $2 h=2 \mathrm{~mm}$. The next section will compare the serrated flat plate with the straight flat plate trailing edge, where the issue of possible noise increase at higher frequency will be addressed.

\section{B. Effect of serration amplitude $(2 \mathrm{~h})$ on the broadband noise reduction}

Figure 5 shows the comparison of the noise spectra for the NACA65(12)-10 aerofoil between a straight (baseline) flat plate and a serrated flat plate trailing edge at $U=24 \mathrm{~ms}^{-1}$, AoA $=0^{\circ}, \lambda=3.3 \mathrm{~mm}$ and $\phi=0^{\circ}$. As discussed previously, the serrated flat plate with $2 h=H$ is compared against a straight flat plate with $2 h=H / 2$, i.e. both have the same wetted area.

For the serrated flat plate that has a low $2 h(\leq 12 \mathrm{~mm})$, the reduction in broadband noise seems to confined to the high frequency end: $1.5 \leq f \leq 5.5 \mathrm{kHz}$ only. At moderately high $2 h \sim 20-30 \mathrm{~mm}$, the reduction in noise "shifts" to the low frequency region of $250 \leq f \leq 1000 \mathrm{~Hz}$, where the reduction of broadband noise at $1.5 \leq f \leq 5.5 \mathrm{kHz}$ becomes less effective. Examination of Fig. 4a, as well as Fig. 5 on the noise spectra produced by the straight flat plate only, suggests that two broadband noise sources become increasingly prominent as the $2 h$ increases: one pre-dominantly at less than $1 \mathrm{kHz}$, and another one occurs between 2 and $5 \mathrm{kHz}$. Noise increase (up to $-2 \mathrm{~dB}$ ) as the consequence of interaction between the cross jet and sawtooth gap is found to occur beyond $6 \mathrm{kHz}$ for this particular velocity $(U=$ $\left.24 \mathrm{~ms}^{-1}\right)$.

The results in Fig. 5 suggest that the serrated flat plate with a low $2 h$ is more effective to reduce the high frequency broadband noise source, while the opposite is true for a moderately high $2 h$. The exception is when $2 h=$ $35 \mathrm{~mm}$ for the serrated flat plate, where it seems to be effective on reducing both noise sources. This particular

4

American Institute of Aeronautics and Astronautics 
configuration $(\lambda=3.3 \mathrm{~mm}, 2 h=35 \mathrm{~mm} \rightarrow \lambda / h=0.19)$, therefore, is chosen for a further investigation on the sensitivity of flap angles to the aerofoil self-noise.

So far, all the analysis focused on a single velocity only. It would be useful to examine the effectiveness of the serrated flat plate trailing edge at higher velocities. Figure 6 shows the difference in sound pressure level, $\Delta$ SPL, between the straight flat plate trailing edge $(2 h=17.5 \mathrm{~mm})$ and the serrated flat plate trailing edge $(\lambda=3.3 \mathrm{~mm}, 2 \mathrm{~h}$ $=35 \mathrm{~mm}$ ) as functions of frequency and velocity. A positive value of $\triangle$ SPL denotes noise reduction, and the opposite is true. It can be seen that this type of serrated flat plate trailing edge is very effective in the reduction of broadband self-noise across a wide velocity range, where $\triangle$ SPL up to $8 \mathrm{~dB}$ can be achieved. At $U \geq 30 \mathrm{~ms}^{-1}$, the frequency bandwidth of the positive $\Delta$ SPL becomes narrower from the high frequency end. This indicates that either the high frequency broadband noise source is weakened, or the serrated flat plate becomes less effective in the reduction of this broadband noise source when the velocity increases. However, the serrated flat plate is consistently effective in the reduction of the low frequency broadband noise source throughout the velocity range investigated here.

Another interesting point to note is the very high frequency noise increase (different to the high frequency broadband noise described earlier). The characteristic frequency of the negative $\Delta$ SPL is found to increase with $U$. This indicates that the noise source is likely to be Strouhal-number dependent on a characteristic length scale chosen to be the turbulent boundary layer thickness in Gruber et $\mathrm{al}^{9}$, as shown in Fig. $3 \mathrm{~b}$ here.

\section{Serrated Trailing Edge with Non-Zero Flap Angles}

This section will investigate the effect of aerofoil self-noise subjected to serrated flat plate with non-zero flap angles. Using the same definition previously, the serrated flat plate of $2 h=35 \mathrm{~mm}$ will be compared against a straight flat plate of $2 h=17.5 \mathrm{~mm}$. This applies to all the non-zero flap angle configurations.

Figure 7a shows a collection of noise spectra produced by straight flat plates for $-15^{\circ} \leq \phi \leq+15^{\circ}$ at $U=24 \mathrm{~ms}^{-1}$ and $\mathrm{AoA}=0^{\circ}$. It is interesting to note that the flap angle does not seem to alter the noise spectra significantly, except for the $\phi=+15^{\circ}$ case (also for the $\phi=+10^{\circ}$ case to a certain extent) where a much larger level of noise radiation is observed at low frequency below $200 \mathrm{~Hz}$. The corresponding $\triangle$ SPL spectra in Fig. 7c mostly demonstrate a fluctuation of data around the $\Delta \mathrm{SPL}=0$ line. Note that the $\Delta \mathrm{SPL}$ here is defined as the difference in sound pressure level between the case when $\phi=0^{\circ}$ (non-flap case), and another case when $\phi \neq 0^{\circ}$. Nonetheless, several trends are still discernible. For flap angles of $5^{\circ} \leq \phi \leq 10^{\circ}, \Delta \mathrm{SPL} \approx+1 \mathrm{~dB}$ can be achieved at $1 \leq f \leq 3 \mathrm{kHz}$, whereby at $f \geq 4$ $\mathrm{kHz}, \Delta \mathrm{SPL} \approx+0.5 \mathrm{~dB}$. For all the $-\phi$ cases, as well as at $\phi=15^{\circ}$, the $\Delta \mathrm{SPL}$ are slightly negative, but not too significantly.

Figure 7b shows a collection of noise spectra produced by serrated flat plates for $-15^{\circ} \leq \phi \leq+15^{\circ}$, and Fig. $7 \mathrm{~d}$ shows the corresponding $\Delta$ SPL spectra. The large noise increase below $200 \mathrm{~Hz}$ by the straight flat plate at $\phi=+15^{\circ}$ is also produced by the serrated flat plate with the same flap angle. This indicates that this low frequency noise increase is caused by the global effect of blade loading at this large positive flap angle.

The serrated flat plates are more sensitive to the flap angles in the aerofoil self-noise radiation. Previously, we have observed that a slight benefit in achieving the low-noise radiation is related to $+5^{\circ} \leq \phi \leq+10^{\circ}$ for the straight flat plate. Similar scenario also applies to the serrated flat plate case, where the $\phi=+5^{\circ}$ covers the largest frequency range of $+\Delta \mathrm{SPL}$, followed by $\phi=+10^{\circ}$ and then $\phi=+15^{\circ}$. In contrast, all the serrated flat plates with negative flap angles consistently under-perform $(-\Delta \mathrm{SPL})$ throughout the frequency range.

\section{A. Effect of serration flap angles $(\phi)$ on the broadband noise reduction}

When it comes to the self-noise radiation, the serrated flat plate is relatively more sensitive to the flap angles than the straight flat plate. This section will present results for the $\Delta$ SPL between a straight flat plate $(2 h=17.5$ $\mathrm{mm})$, and a serrated flat plate $(2 h=35 \mathrm{~mm})$ for $-15^{\circ} \leq \phi \leq+15^{\circ}$ at $U=24 \mathrm{~ms}^{-1}$ and AoA $=0^{\circ}$. Figure 8a compares the $\triangle$ SPL spectra for all negative flap angle, $\phi=-15^{\circ},-10^{\circ}$ and $-5^{\circ}$, including the $\phi=0^{\circ}$ non-flap case as the reference. In general, $+\Delta \mathrm{SPL}$ starts to occur at $100 \mathrm{~Hz}$, and up to between $4.3 \mathrm{kHz}$ (for the $\phi=-5^{\circ}$ case) and $6.0 \mathrm{kHz}$ (for the non-flap case). In this frequency range for the $+\Delta \mathrm{SPL}$, the best noise performance is achieved by the nonflap case, followed by $\phi=-15^{\circ}$ and then the $\phi=-10^{\circ}$ cases. The $\phi=-5^{\circ}$ case produces the lowest level of $+\Delta \mathrm{SPL}$ over a narrower frequency range. Its performance index is exacerbated when examining at $f \geq 4.3 \mathrm{kHz}$, where it produces the highest $-\Delta$ SPL level amongst others. Following a similar trend, the $\phi=-10^{\circ}$ produces the second 
highest $-\Delta$ SPL, followed by the $\phi=-15^{\circ}$, where the non-flap case produces the lowest. A summary that can be drawn in Fig. 8a is that the noise performance of a serrated flat plate with a large negative flap angle is not too different with the non-flap case, but as the negative flap angle reduces, the noise performances deteriorates.

Figure 8b compares the $\Delta$ SPL spectra for all positive flap angle, $\phi=+15^{\circ},+10^{\circ}$ and $+5^{\circ}$, again with the non-flap case $\phi=0^{\circ}$ spectrum included. At a small positive flap angle of $\phi=+5^{\circ}$, the corresponding broadband noise reduction $(+\Delta$ SPL) at $150 \leq f \leq 740 \mathrm{~Hz}$ is $2 \mathrm{~dB}$ lower than the non-flap case. At $740<f \leq 1660 \mathrm{~Hz}$, both spectra exhibit a similar level of $+\Delta \mathrm{SPL}$. At $f>1660 \mathrm{~Hz}$, the $+\Delta \mathrm{SPL}$ achieved by the $\phi=+5^{\circ}$ consistently out-performs the non-flap case (up to $1.5 \mathrm{~dB}$ higher), even surpassing the very high frequency zone where the dominant noise source is the "leakage" noise by the cross-jet through the sawtooth gaps.

By ignoring the results at less than $200 \mathrm{~Hz}$, noise reduction only starts to occur from $400 \mathrm{~Hz}$ when the positive flap angle is increased to either $\phi=+10^{\circ}$ or $+15^{\circ}$. At this point, the non-flap case has already achieved an $\Delta$ SPL of $+5.3 \mathrm{~dB}$. The $+\Delta$ SPL produced by the $\phi=+10^{\circ}$ or $+15^{\circ}$ exhibit a slow recovery when the frequency is increased, and they eventually intersect with the non-flap case at $2.1 \mathrm{kHz}$. Above this frequency, the $\phi=+10^{\circ}$ or $+15^{\circ}$ produces the best noise performance that surpasses both the non-flap and low positive flap angle $\left(\phi=+5^{\circ}\right)$ cases.

Another point that is worth mentioning is that, at very high frequency $f>10 \mathrm{kHz}$, the $\phi=+15^{\circ}$ achieves a predominantly $+\Delta \mathrm{SPL}$, indicating that the leakage noise is suppressed in this case.

A summary that can be drawn in Fig. $8 \mathrm{~b}$ is that the serrated flat plate with a small positive flap angle $\left(\phi=+5^{\circ}\right)$ performs better at low frequency, but less so at high frequency when it is compared against the same serrated flat plate but at larger positive flap angles. The non-flap case only achieves a marginally better noise reduction than the $\phi=+5^{\circ}$ case at the low frequency between $150 \leq f \leq 740 \mathrm{~Hz}$. At higher frequencies, however, the non-flap case is consistently out-performed by the $\phi=+5^{\circ}$ case.

\section{B. Boundary layer and wake flow produced by the serrated trailing edge at $\phi=-10^{\circ}, 0^{\circ}$, and $+10^{\circ}$}

In order to shed some lights on the hydrodynamic fields pertaining to the serrated aerofoil self-noise with flap angles, some boundary layer and wake flow measurements were performed in the Brunel aeroacoustic facility under the same flow setting as the noise tests. A schematic to illustrate the experimental set up for the flow measurements is shown in Fig. 9. The boundary layer measurements were performed at both the suction surface and pressure surface. The measurement point at each surface is always situated at the same location, i.e. $5 \mathrm{~mm}$ upstream of the interface between the aerofoil and the cardboard flat plate (see Fig. 9). We also ensure that the spanwise (z) location of the boundary layer measurement point coincides with the location of one of the sawtooth tip. Each boundary layer profile contains 46 points, with a finer spatial resolution at the near wall region $(\Delta y \approx 0.05 \mathrm{~mm})$. The nearest measurement point with relative to the aerofoil surface is about $0.5 \mathrm{~mm}$.

Also shown in Fig. 9 is the set up for the measurement of the near wake turbulent velocity by two single hot wire probes - one is termed as the "stationary" probe, and another is called the "traversing" probe. The use of two hot wire probes is to determine the spanwise coherence of the near wake turbulent velocity, $\gamma^{2}$. The spanwise coherence $\gamma^{2}$ is defined as:

$$
\gamma^{2}=\frac{\left|\Phi_{v_{i} v_{j}}(f)\right|^{2}}{\Phi_{v_{i} v_{i}}(f) \Phi_{v_{j} v_{j}}(f)},
$$

where $0 \leq \gamma^{2} \leq 1 . \Phi_{v_{i} v_{j}}(f)$ is the cross-spectrum between the two streamwise fluctuating velocity signals $v_{i}$ and $v_{j}$. The velocity measurement $v_{i}$ was measured by the "stationary" probe downstream of the sawtooth tip at $y=0$. The velocity measurement $v_{j}$ was measured by the "traversing" probe situated at the same downstream position but was traversed along the spanwise $\Delta z$ direction. $\Phi_{v_{i} v_{i}}(f)$ and $\Phi_{v_{j} v_{j}}(f)$ are the autospectra of each individual fluctuating velocity signals.

Note that for both the boundary layer and near wake measurements, the experiment was carried out at exactly the same flow condition as the previous noise test: $U=24 \mathrm{~ms}^{-1}$ and AoA $=0^{\circ}$. Three flap angle cases were investigated: $\phi=-10^{\circ}$ (flap-down), $0^{\circ}$ (non-flap) and $+10^{\circ}$ (flap-up).

The results from the boundary layer measurements were first discussed. Figure 10a shows the boundary layer mean velocity profiles at the suction and pressure surfaces for the three flap angle cases for the baseline, straight flat plate. Note that the freestream velocity, $u_{\infty}$ is taken as the velocity measured at the largest $y$ location away from the aerofoil surface $(\sim 25.5 \mathrm{~mm})$. Boundary layer thickness developed at the pressure surface is considerably larger than that developed at the suction surface. This might be due to the NACA65(12)-10 being designated as a laminar 
aerofoil, where the highest point at the suction side occurs at considerable distance downstream of the leading edge (see Fig. 1). Therefore, the growth of boundary layer on the suction surface undergoes a considerable acceleration over the first half of the aerofoil, before slowly diffuse towards the trailing edge in the adverse pressure gradient over the rear half. On the other hand, the pressure surface is largely flat and there is minimal external pressure force acting on the boundary layer of the pressure surface, where it can grow considerably by the time it reaches the trailing edge.

When a negative flap angle is used $\left(\phi=-10^{\circ}\right)$, the boundary layer at the suction surface becomes fuller, and even the $u / u_{\infty}$ at $3 \leq y \leq 19 \mathrm{~mm}$ has been found to exceed unity. This means that the velocity at this region is higher than the freestream value, suggesting the presence of wall-jet liked phenomenon. The boundary layer thickness is also thinner than that of the non-flap case. By examining the profile at the pressure surface at the same flap angle, the boundary layer becomes less full and the thickness is clearly thicker than the non-flap case. These observations can be corroborated in the corresponding turbulent velocity profiles in Fig. 10b. Compared to the non-flap case, the turbulence intensity across the thinner boundary layer at the suction surface becomes lower, whilst the turbulence intensity across the thicker boundary layer at the pressure surface becomes larger. Physically, deploying a flap-down of the straight flat plate will enhance the downwash to stabilise the boundary layer near the trailing edge at the suction surface, while it will increase the turbulence characteristics at the pressure surface near the trailing edge.

When a positive flap angle is used $\left(\phi=+10^{\circ}\right)$, a completely opposite trend is observed. The flap-up of the straight flat plate is shown to thicken the boundary layer and increase the overall turbulence intensity at the suction surface, but the boundary layer at the pressure surface becomes thinner and the overall turbulence intensity is lower.

The analysis now focuses on the comparison between the straight flat plates and serrated flat plates at different flap angles. Any changes in the boundary layer velocity profile can be solely attributed to the sawtooth serration geometry. As shown in Fig. 11a on the suction surface, the wall-jet phenomenon previously observed in the straight flat plate with flap-down no longer exists when a serrated flat plate, also at the same flap-down position, is used. This implies that the stabilising effect of the flap-down has been weakened by the presence of sawtooth air gap in the serrated flat plate. Similar trend is also observed for the non-flap case. However, the boundary layer profiles for the straight flat plate and serrated flat plate, where both are at the same flap-up position, do not exhibit significant difference with each other.

In Fig. $11 \mathrm{~b}$ on the pressure surface, again the boundary layer profiles for the straight flat plate and serrated flat plate do not exhibit significant difference with each other when they are both at the flap-up position. At the non-flap position, the serrated flat plate becomes thinner and fuller than the straight flat plate counterpart. Similar trend is also observed for the flap-down case.

To summarise the boundary layer results, for non-flap and flap-down positions, a serrated flat plate can alter the boundary layer properties near the suction side and pressure side trailing edges in an opposite manner: stabilise the boundary layer at the pressure surface, and opposite is true for the suction surface boundary layer. However, at flapup position, the boundary layer properties seem to be insensitive to the serrated flat plate.

Now, the analysis will focus on the near wake region. The near wake power spectral density of the fluctuating velocity across the $\Delta z$ at the trailing edge tip ( $y=0$, see definition in Fig. 9) is shown in Fig. 12a. Note that $\Delta z=0$ corresponds to the location of the "stationary" probe that coincides with the first sawtooth tip. Therefore, all the data presented in Fig. 12a is measured by the "traversing" probe. For the straight flat plate in the flap-down, non-flap and flap-up configurations, the wake $P S D$ are constant across the spanwise direction. However, for the serrated flat plate at the flap-down and non-flap configurations, a clear periodic vortex shedding PSD is displayed.

For the flap-down case of the serrated flat plate, the $P S D$ level is minimum at the sawtooth tip, but achieves maximum at the oblique edges close to the tip. This resembles the oblique vortical structures that are pre-dominant at the oblique edges of the sawtooth. Interestingly, an cross-jet initiated by these vortical structures have been shown to enhance the interaction with the sawtooth structure to cause an increase of the high frequency noise (see Fig. 8a). For the non-flap case of the serrated flat plate, the PSD maxima occur at the sawtooth root, slightly different with the flap-down case. Structural interaction of the vortical structure might be less intense in this case since the main vortical structure is generated in the middle of the sawtooth gaps. This is also reflected in Fig. 8a where the level of noise increase at high frequency is less than that generated by the flap-down case. For the flap-up case of the serrated flat plate, interestingly, the $P S D$ spectrum at the near wake is not much different with the PSD spectrum generated by the straight flat plate. It is conjectured that the main wake is generated at a $y$ location close to the sawtooth root, not at the sawtooth tip where the wake measurement took place in this flap-up case. In other words, the boundary layer near the trailing edge bypasses the sawtooth gaps and sheds into the shear layers directly. Because of the lack of activity in cross jet within the sawtooth, noise increase at high frequency is not apparent (see Fig. 8b). 
The presence of a vortical structure can also be examined from the coherence spectra in Fig. 12b. For all the straight flat plate in the flap-down, non-flap and flap-up cases, relatively large spanwise coherence is only observed for $\Delta z$ up to approximately $0.7 \mathrm{~mm}$. This indicates that the coherence is mostly related to the turbulent boundary layer structures.

For the serrated flat plate at flap-down configuration, however, the largest spanwise coherence is achieved near the second sawtooth tip $(\Delta z \approx 3.3 \mathrm{~mm})$. Within the error of margin, this relatively high spanwise coherence is most likely to be associated to the oblique vortical structure identified earlier. This also suggests that the source of these oblique vortical structures is the same for each consecutive sawtooth. Similar picture can be drawn for the serrated flat plate with a non-flap configuration. However, no obvious spanwise coherence related to the vortical structure can be found for the serrated flat plate at the flap-up configuration.

\section{Conclusion}

The serration amplitude and serration wavelength are traditionally regarded as the primary geometrical variables that can affect the noise performance of an add-on, flat plate type serrated trailing edge. This experimental study investigates another serration geometrical variable, namely the serration flap angle that could potentially affect the self-noise reduction of an aerofoil. The experiment was carried out at Brunel aeroacoustic facility, on a NACA65(12)-10 aerofoil. The serrated flat plates were manufactured to form in several flap angles: $\pm 15^{\circ}, \pm 10^{\circ}, \pm 5^{\circ}$ and $0^{\circ}$ as the reference.

Preliminary investigation on the effect of serration amplitude, without the flap angle, confirms with other findings that the largest level of broadband noise reduction is achieved when the amplitude of the serrated flat plate is large. It is also worth reporting that broadband noise can already be reduced even by attaching a large chord length of unserrated, straight flat plate. When the serrated flat plate contains a flap angle, it is generally observed that a flap-up position (positive flap angle) is more favourable for broadband noise reduction, while the opposite is true for the flap-down position (negative flap angle). The best flap-up position is when the positive flap angle is small, at around $+5^{\circ}$. Unfortunately, a small flap-down position, i.e. $-5^{\circ}$ is the worst performer amongst the test cases (lowest level of broadband noise reduction at low frequency, and highest noise increase at high frequency). Therefore, even a small misalignment of the trailing edge serration due to the manufacturing defect could potentially degrade (or enhance) the overall aerofoil self-noise reduction because the serration is found to be sensitive to small flap angles.

\section{Acknowledgment}

The authors would like to thank the discussions with Jeremy Hurault and Mohammad Kamruzzaman from the Vestas Wind System about the serration flap angle.

\section{References}

1 A. Wolf, T. H. Lutz, W. Wurz, E. Kramer, O. Stalnov and A. Seifert, "Trailing edge noise reduction of wind turbine blades by active flow control," Wind Energ 18, 909 (2015).

2 A. Inasawa, C. Ninomiya and M. Asai, "Suppression of tonal trailing-edge noise from an airfoil using a plasma actuator," AIAA J 51, 1695 (2013).

O. Rodriguez, "Base drag reduction by control of the three-dimensional unsteady vortical structure," Exp Fluids 11, 218 (1991).

A. M. Knepper, "Examination of three candidate technologies for high-lift devices on aircraft wing," Ph.D. thesis, Cranfield University, 2005.

M. S. Howe, "Aerodynamic noise of a serrated trailing edge," J Fluid Struct 5, 33 (1990).

M. S. Howe, "Noise produced by a sawtooth trailing edge," J Acoust Soc Am 90, 482 (1991).

K. Braun, N. Van der Borg, A. Dassen, F. Doorenspleet, A. Gordner, J. Ocker and R. Parchen, "Serrated trailing edge noise (STENO)," in Proccedings of the European Wind Energy Conference, Nice, France, James \& James (Science Publishers) Ltd., London, U.K., (1999) 180 .

8 S. Oerlemans, M. Fisher, T. Maeder, and K. Kögler "Reduction of wind turbine noise using optimized airfoils and trailing-edge serrations," AIAA J 47, 1470 (2009).

$9 \quad$ M. Gruber, P. F. Joseph, and T. P. Chong, “On the mechanisms of serrated airfoil trailing edge noise reduction,” AIAA Paper No. 20112781, 2011.

10 T. P. Chong, A. Vathylakis, P. F. Joseph, and M. Gruber, "Self-noise produced by an airfoil with nonflat plate trailing-edge serrations," AIAA J 51, 2665 (2013).

11 J. Hurault, A. Gupta, E. Sloth, N. C. Nielsen, A. Borgoltz and P. Ravetta, "Aeroacoustic wind tunnel experiment for serration design optimisation and its application to a wind turbine rotor," In $6^{\text {th }}$ International Meeting on Wind Turbine Noise, Glasgow (2015).

12 R. Amiet, "Noise due to turbulent flow past a trailing edge," J Sound Vib 47, 387 (1976). 

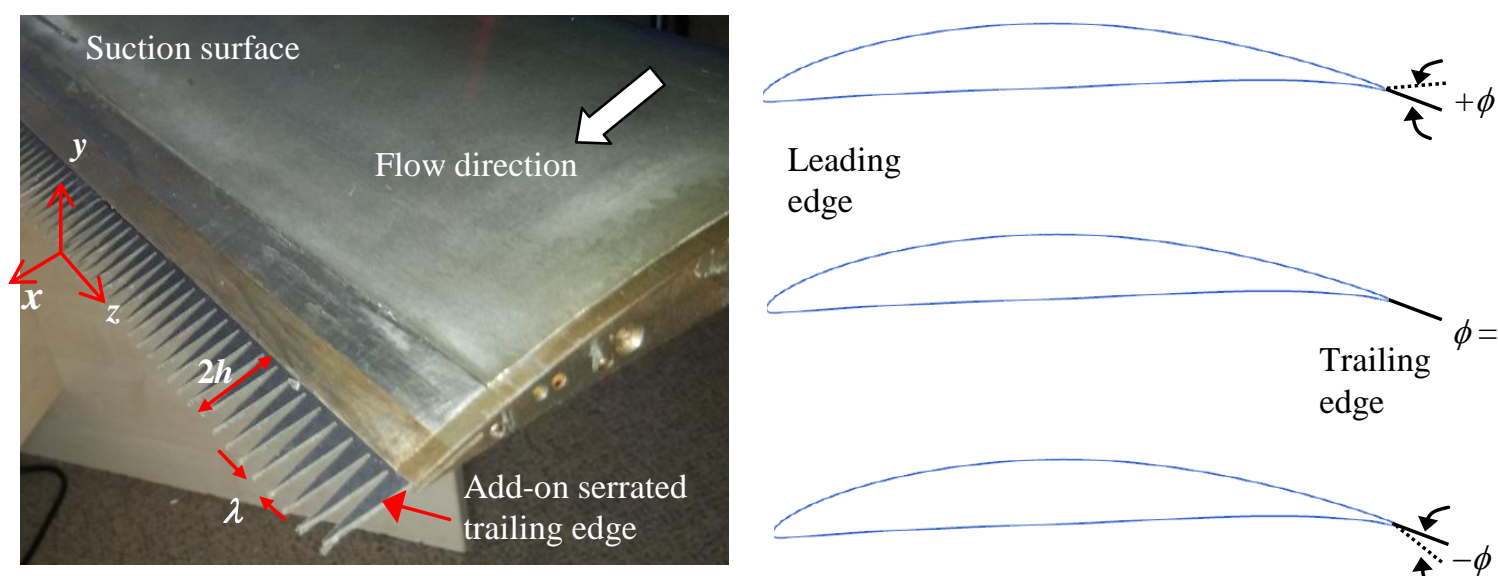

\section{Leading}

edge

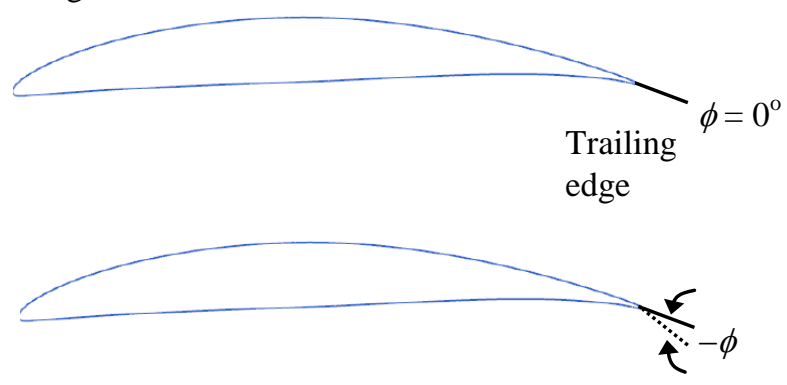

Fig. 1 Parameters associated with an add-on serrated sawtooth geometry on a NACA65(12)-10: serration length $(2 h)$, serration wavelength $(\lambda)$ and serration flap angle $(\phi)$.

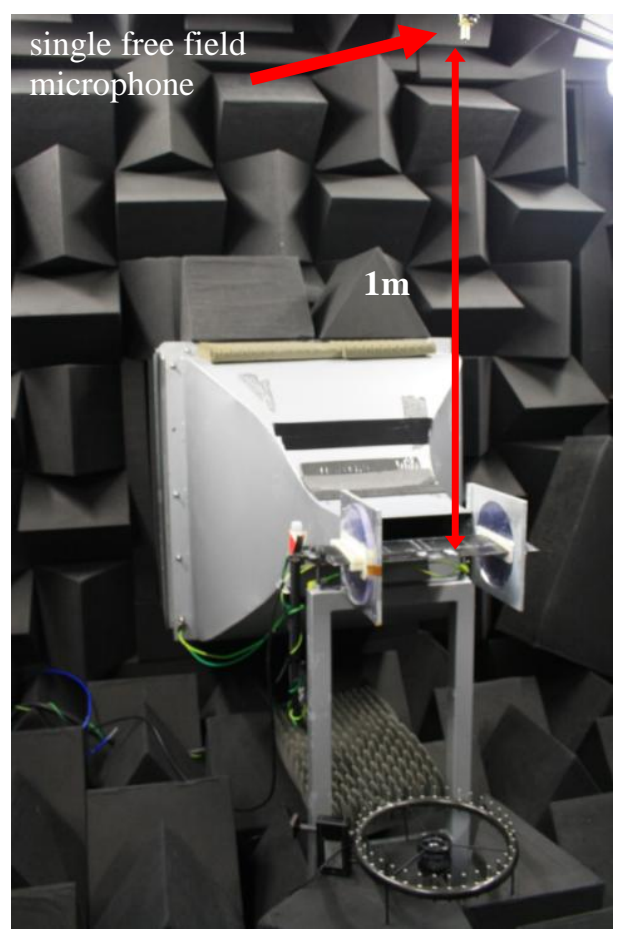

Fig. 2 Experimental set up for the airfoil noise tests in an aeroacoustic wind tunnel facility. 


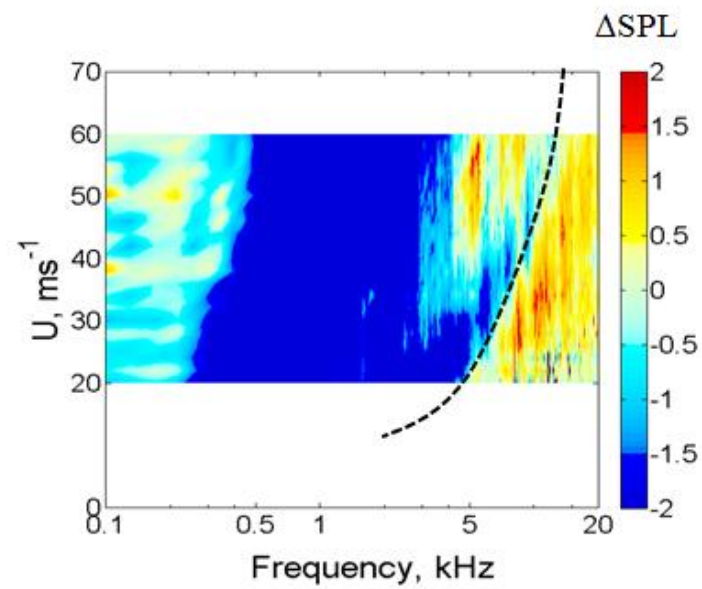

Serration $\lambda / h=0.2,0^{\circ} \mathrm{AoA}$

(a)

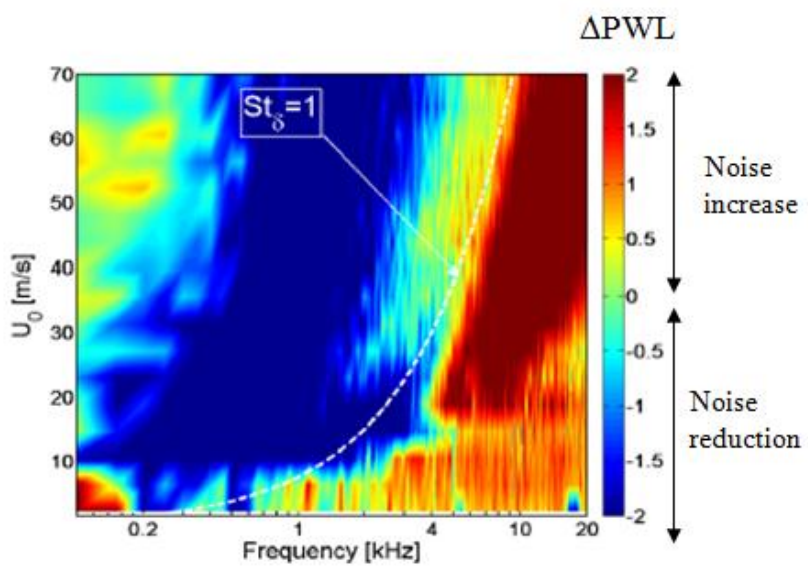

Serration $\lambda / h=0.2,0^{\circ} \mathrm{AoA}$

(b)

Fig. 3 Qualitative comparison between the (a) $\triangle$ SPL obtained at the Brunel facility, and (b) $\triangle$ PWL obtained at the ISVR facility ${ }^{9}$ for the same serrated sawtooth geometry. Note that negative $\triangle$ SPL or $\triangle$ PWL denotes noise reduction, and vice versa. This definition is only applicable in this figure. 
(a)

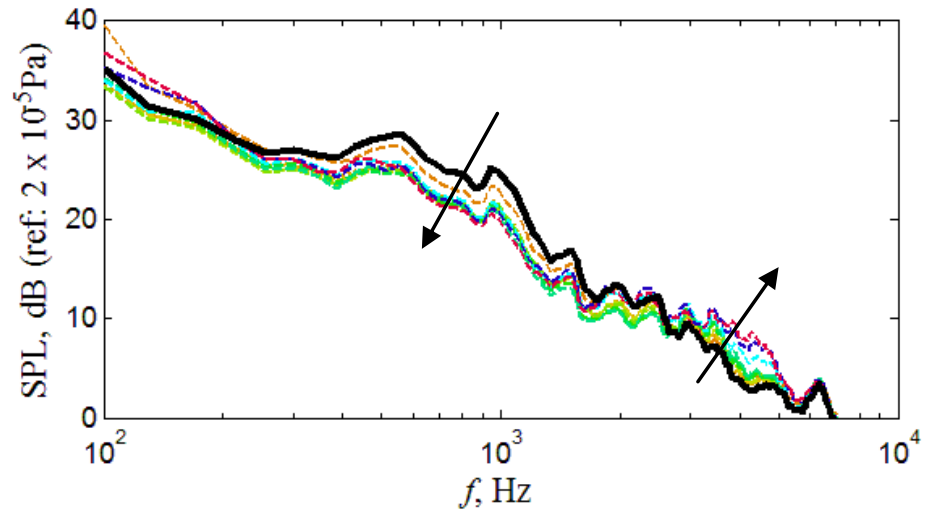

$2 h(\mathbf{m m})$

(b)

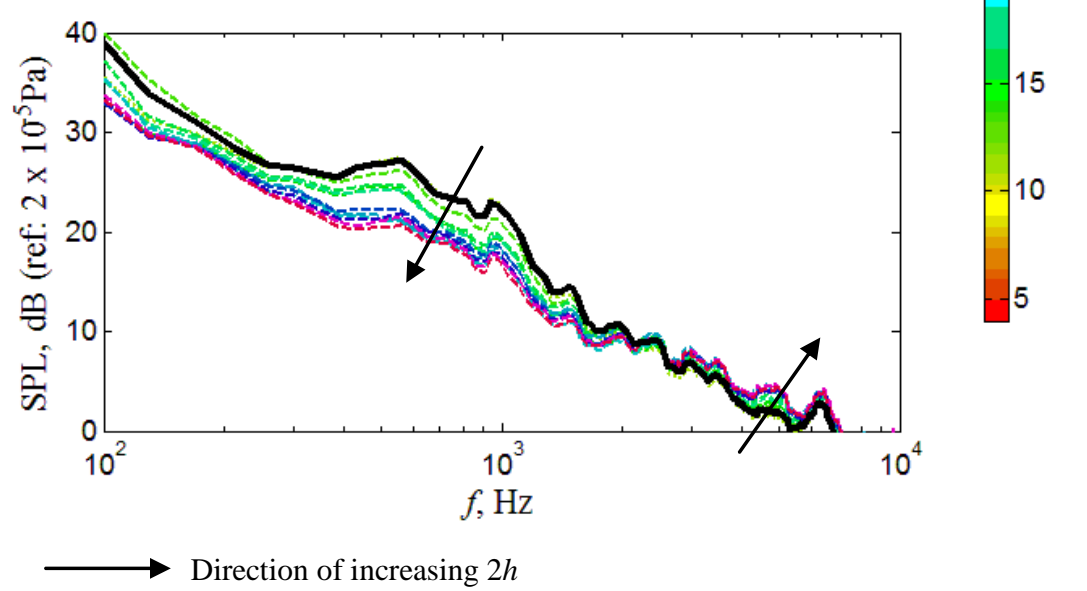

Fig. 4 Noise spectra radiated by the aerofoil installed with (a) straight (baseline) flat plate inserts only, and (b) serrated flat plate inserts only, across a range of $2 \mathrm{~h}$. Note that the serration wavelength for the serrated flat plate is $\lambda=3.3 \mathrm{~mm}$. Both the black lines (-) in (a) and (b) denote noise spectra when $2 h=2 \mathrm{~mm}$ for straight and serrated flat plates, respectively. The jet velocity, $U=24 \mathrm{~ms}^{-1}, \mathrm{AoA}=0^{\circ}$ and flap angle, $\phi=0^{\circ}$. 

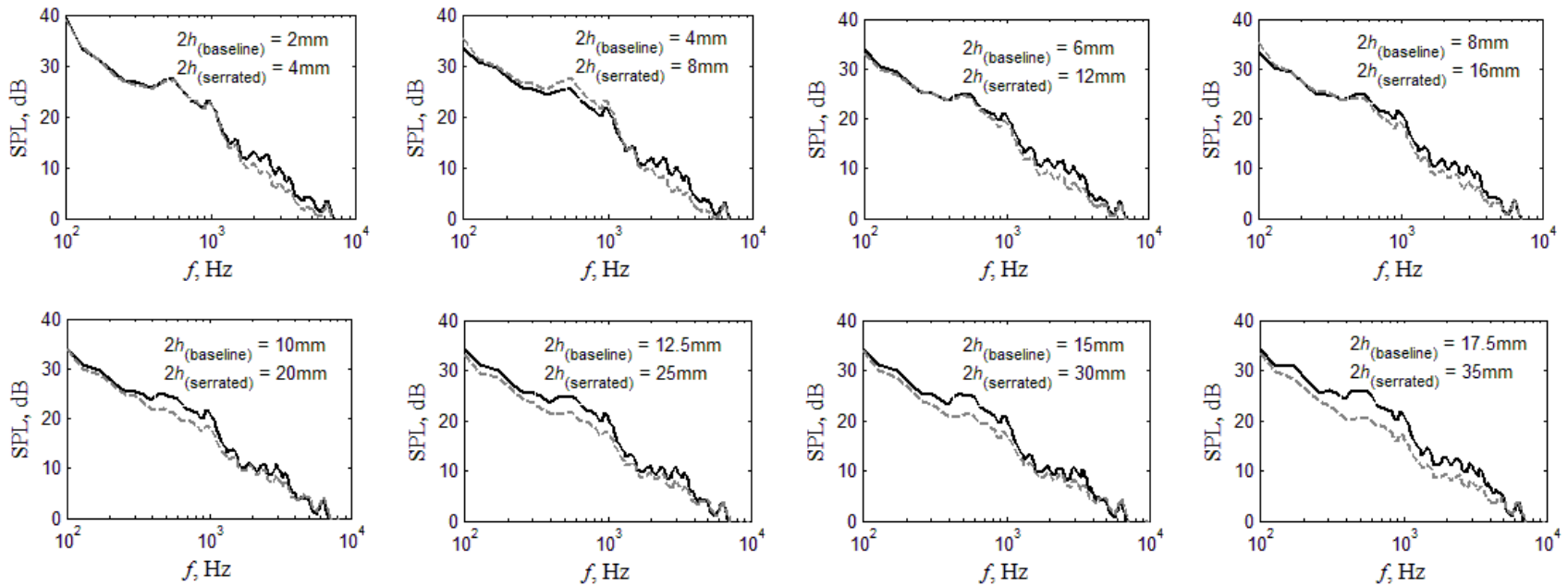

Fig. 5 Comparison of noise spectra radiated by the aerofoil installed with straight (baseline) flat plate inserts (-) and serrated flat plate inserts (- - ) where the $2 h$, respectively, will result in the same wetted area. The jet velocity, $U=24 \mathrm{~ms}^{-1}, \mathrm{AoA}=0^{\circ}$ and flap angle, $\phi=0^{\circ}$. 


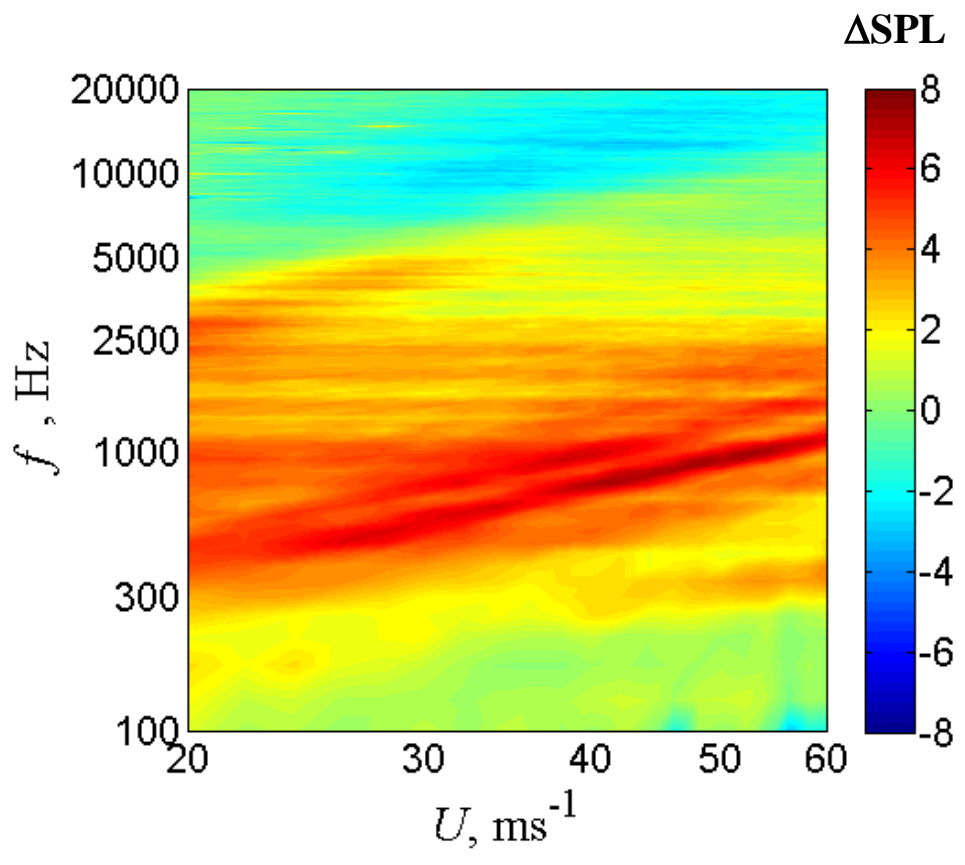

Fig. 6 Contour map of $\Delta \mathrm{SPL}\left(\mathrm{SPL}_{\text {Baseline }}[2 h=17.5 \mathrm{~mm}]\right.$

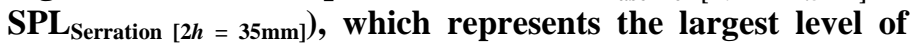
noise reduction achieved in the current study. $A o A=0^{\circ}$ and flap angle, $\phi=0^{\circ}$. Note that $+\Delta$ SPL denotes noise reduction, and vice versa. 
(a)

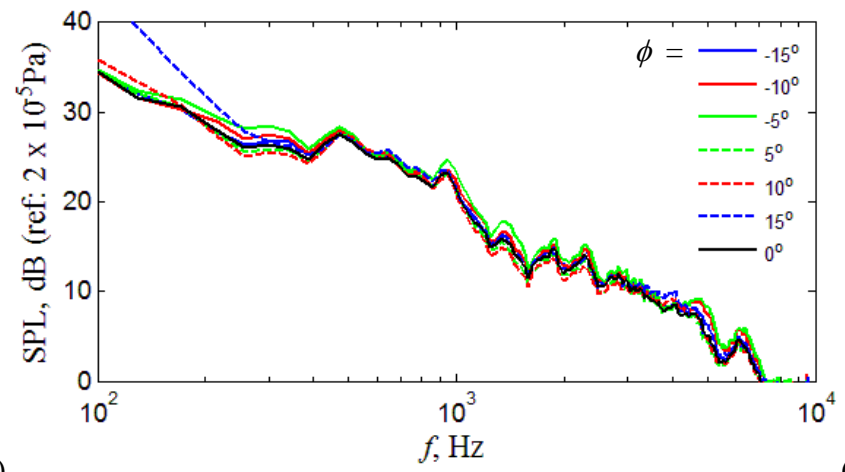

(b)

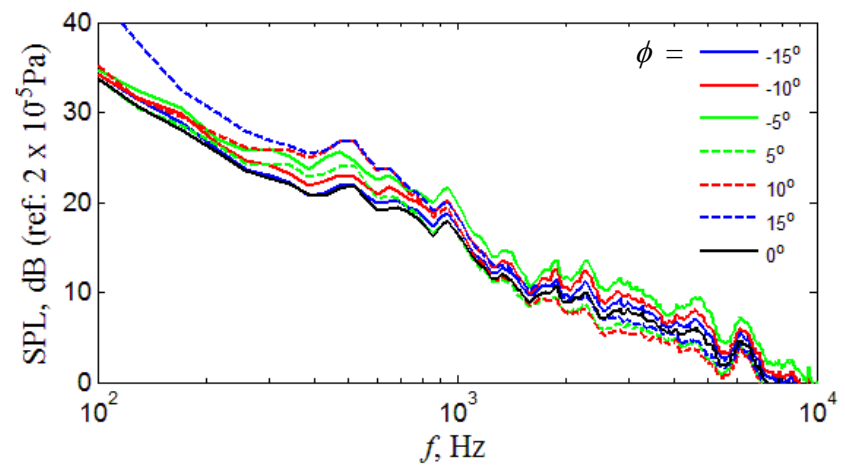

(c)

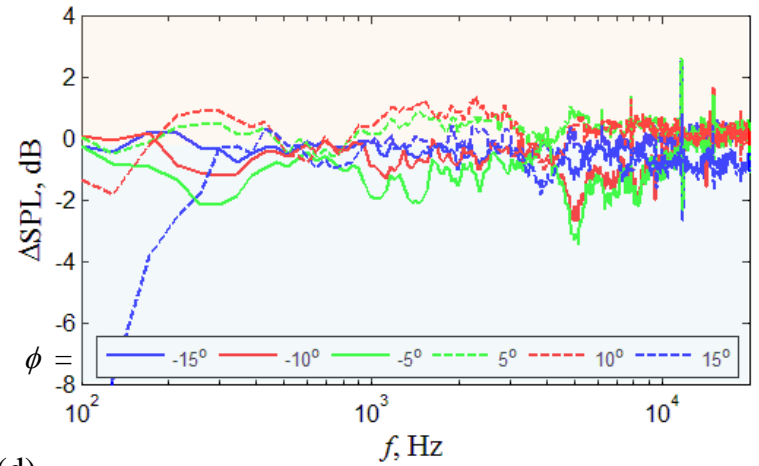

(d)

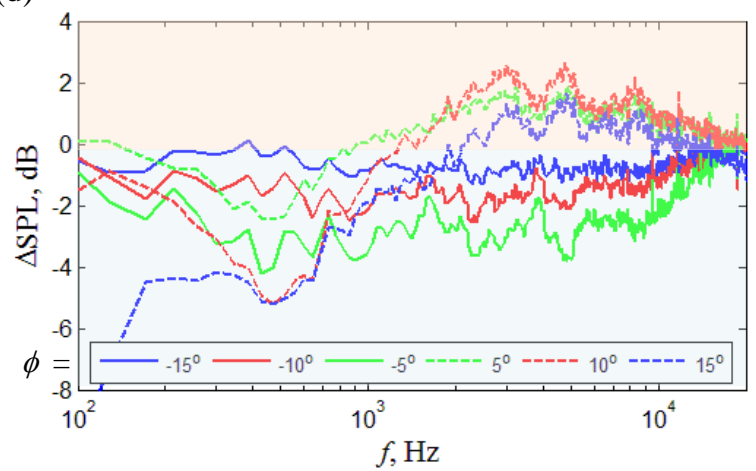

Fig. 7 Comparisons of noise spectra at different flap angles $-15^{\circ} \leq \phi \leq+15^{\circ}$ for (a) straight (baseline) flat plate inserts only, and (b) serrated flat plate inserts only. Sub-figures (c-d) show the $\Delta$ SPL $\left(\mathrm{SPL}_{\left[\phi=0^{0}\right]}-\mathrm{SPL}_{[\phi \neq 0]}\right)$ for straight baseline flap plate insert and serrated flat plate inserts, respectively. The jet velocity, $U=24 \mathrm{~ms}^{-1}$ and $\mathrm{AoA}=0^{\circ}$.

(a)

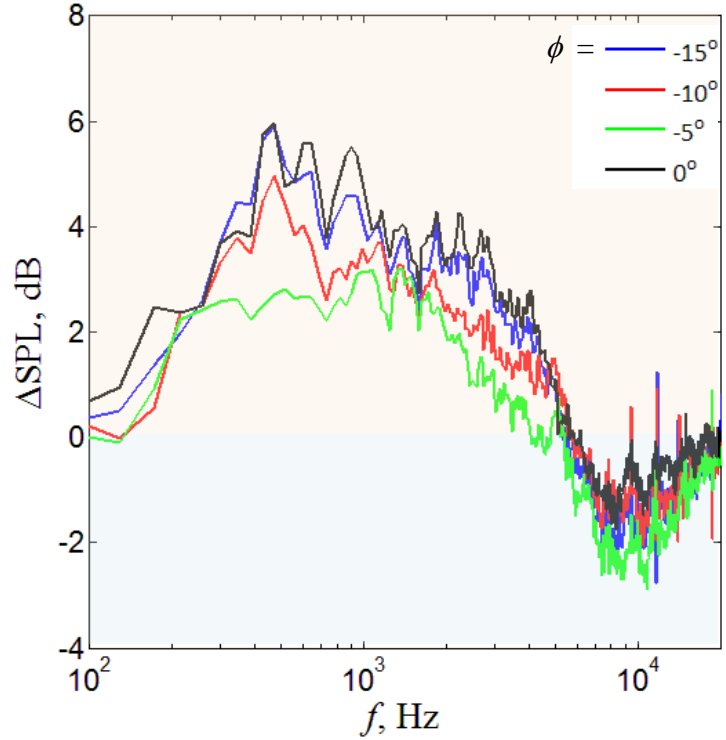

(b)

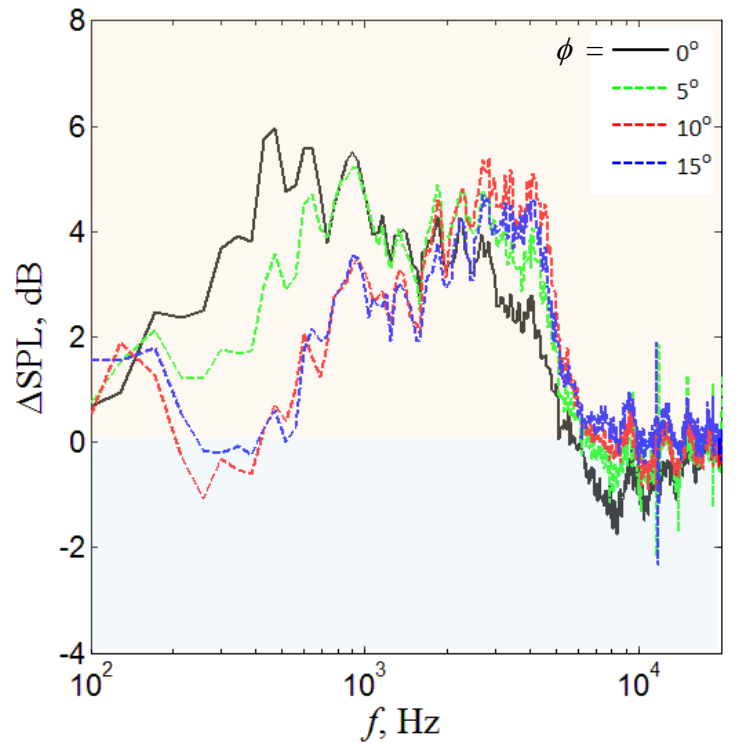

Fig. 8 Comparisons of $\triangle \mathrm{SPL}\left(\mathrm{SPL}_{\mathrm{Baseline}[2 h=17.5 \mathrm{~mm}]}-\mathrm{SPL}_{\mathrm{Serration}[2 h=35 \mathrm{~mm}]}\right)$ at different flap angles: (a) $-15^{\circ} \leq \phi \leq 0^{\circ}$, and (b) $0^{\circ} \leq \phi \leq+15^{\circ}$. The jet velocity, $U=24 \mathrm{~ms}^{-1}$ and $A o A=0^{\circ}$. 

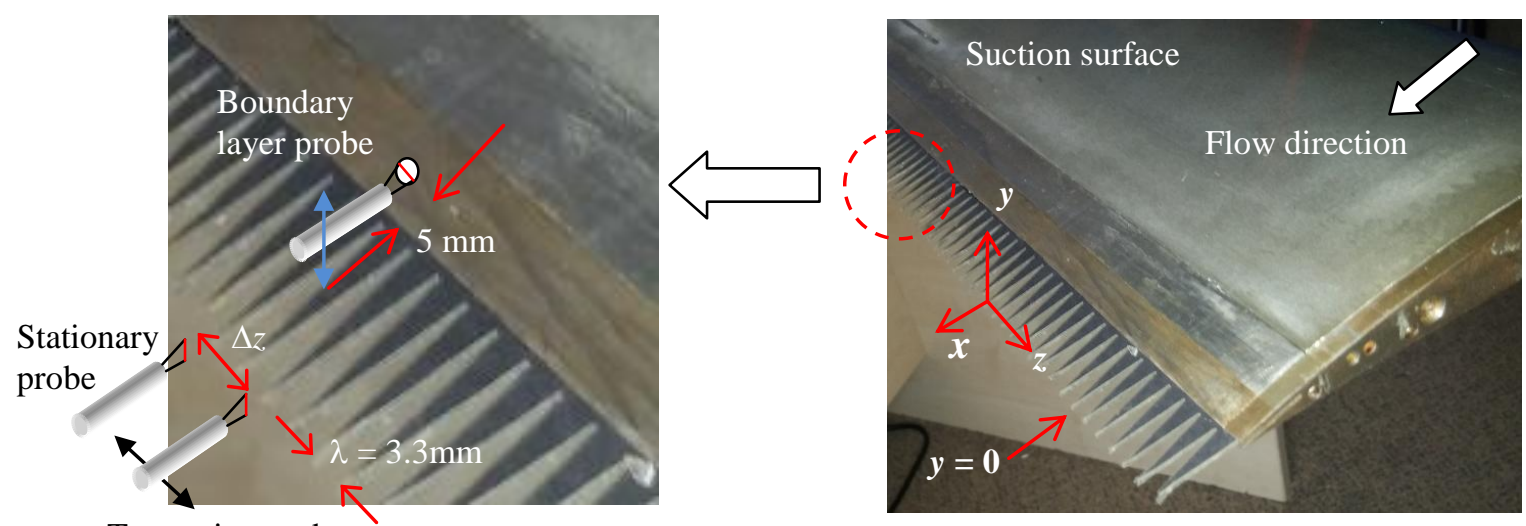

Traversing probe

Fig. 9 Illustration of the measurement locations for the boundary layer properties and near wake velocity power spectral density and coherence.

(a)

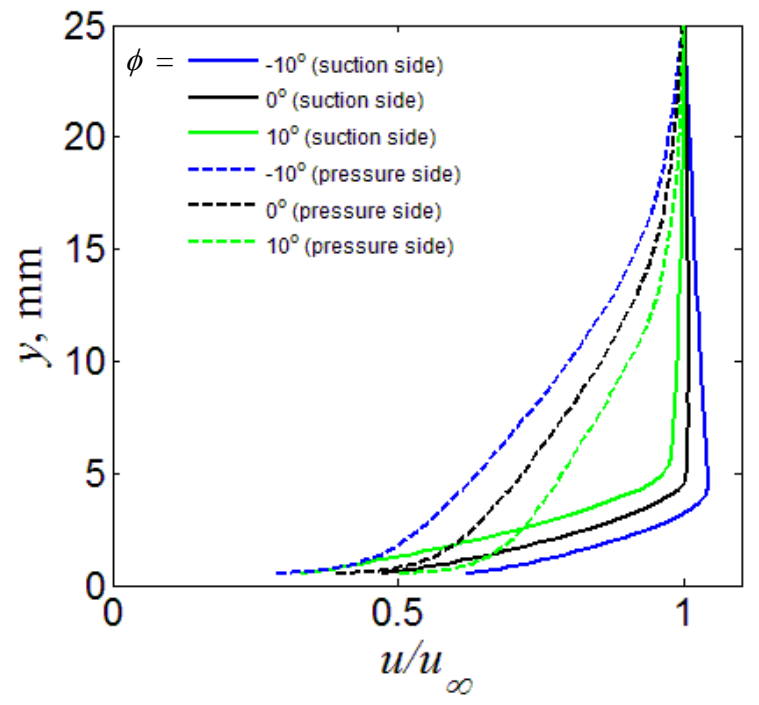

(b)

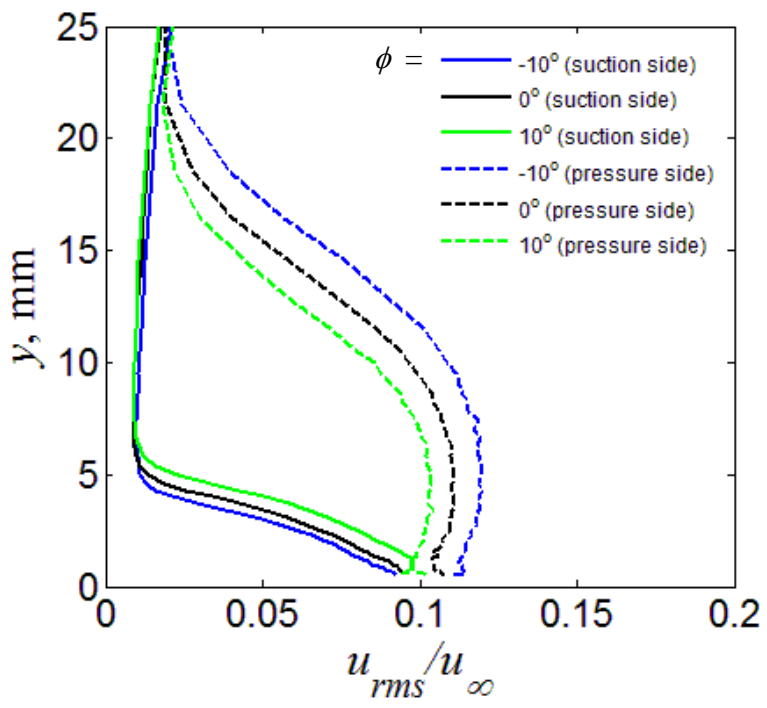

Fig. 10 Comparisons of boundary layer velocity (a) mean profiles, and (b) turbulence profiles at the suction and pressure surfaces subjected to different flap angles, $\phi=-10^{\circ}, 0^{\circ}$ and $10^{\circ}$ for a baseline, straight trailing edge only. The jet velocity, $U=24 \mathrm{~ms}^{-1}$ and $\mathrm{AoA}=0^{\circ}$. 
(a)

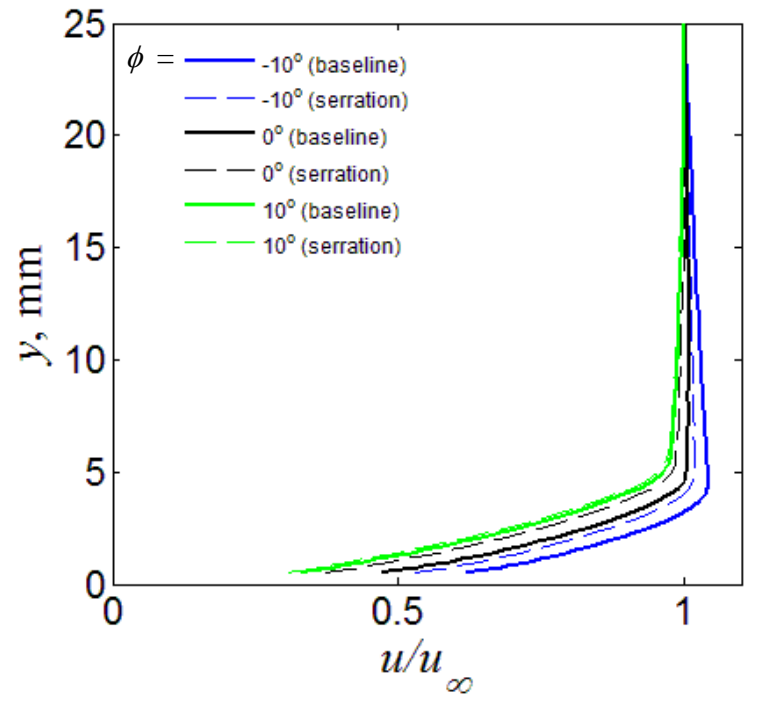

(b)

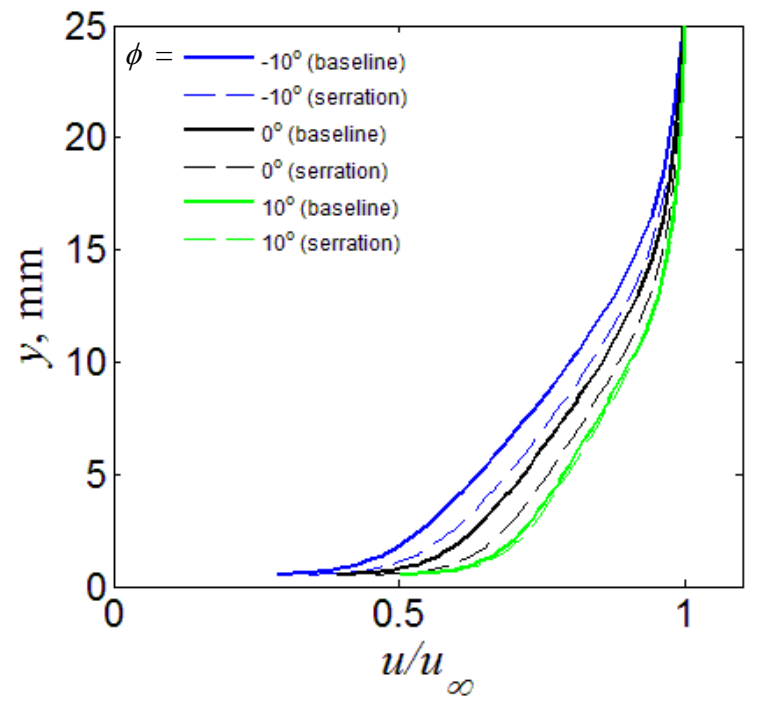

Fig. 11 Comparisons of boundary layer velocity mean profiles at the (a) suction surface, and (b) pressure surface subjected to different flap angles, $\phi=-10^{\circ}, 0^{\circ}$ and $10^{\circ}$ between baseline and serrated trailing edges. The jet velocity, $U=24 \mathrm{~ms}^{-1}$ and $\mathrm{AoA}=0^{\circ}$. 
(a)

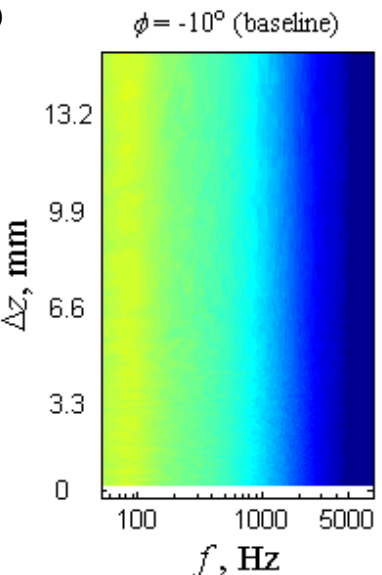

(b)

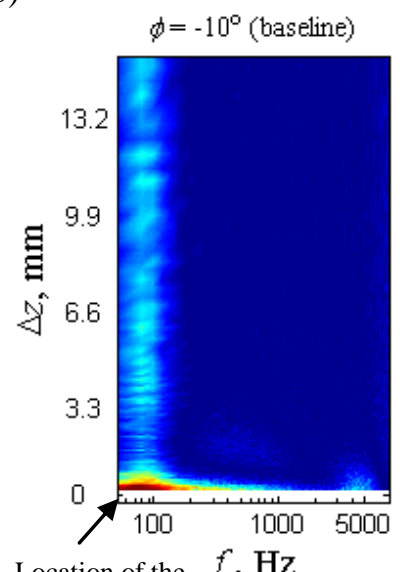

Location of the $f, \mathrm{~Hz}$

stationary probe $\phi=-10^{\circ}$ (serration)
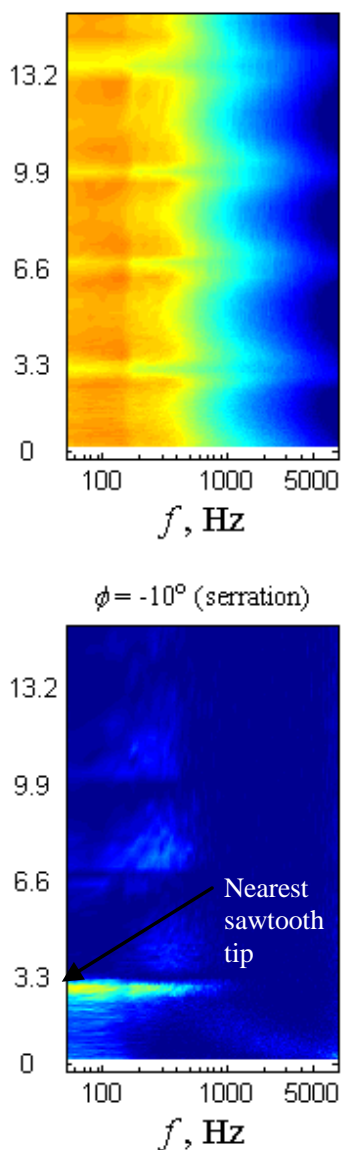

$\phi=0^{\circ}$ (baseline)

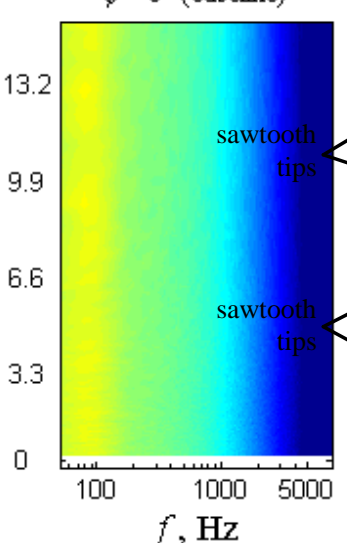

$\phi=0^{\circ}$ (baseline)

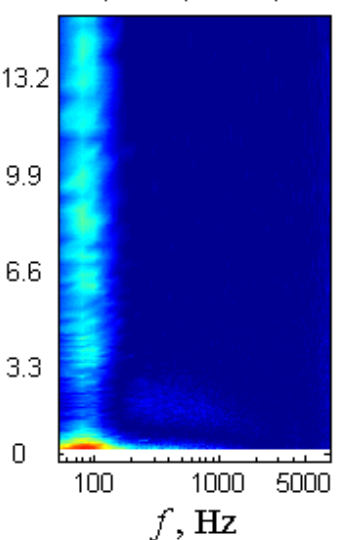

$\phi=0^{\circ}$ (serration)
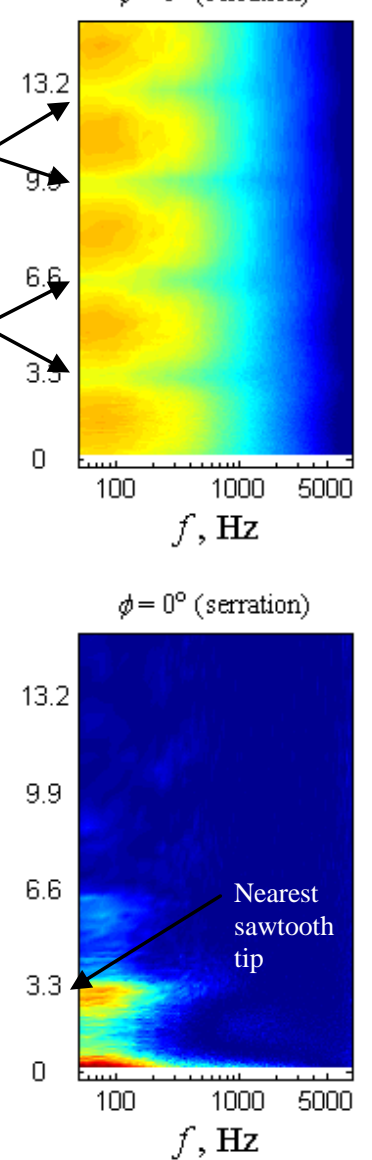
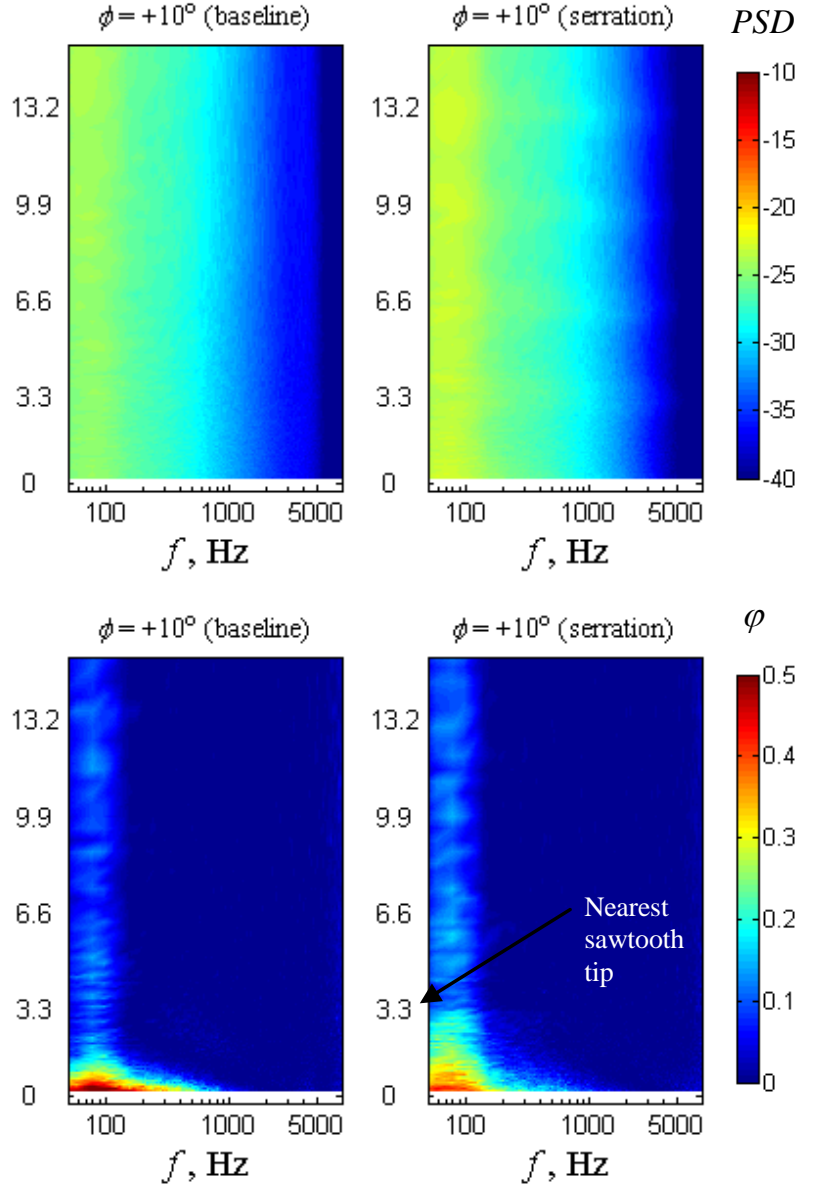

Fig. 12 (a) Fluctuating velocity power spectral density at the near wake as a function of $\Delta z$ at $\mathbf{y}=0 \mathrm{~mm}$ (trailing edge tip), as measured by the traversing probe (see Fig. 9), and (b) coherence function at the near wake as a function of $\Delta z$ at $y=0$ mm (trailing edge tip), as measured by both the stationary and traversing probes (see Fig. 9). Three flap angles, $\phi=-10^{\circ}$, $0^{\circ}$ and $10^{\circ}$ between baseline and serrated trailing edges were investigated. The jet velocity, $U=24 \mathrm{~ms}^{-1}$ and $A o A=0^{\circ}$. 